\title{
Análisis del desarrollo de competencias en la formación inicial del profesorado de Educación Secundaria en España
}

\author{
Guillermo Domínguez Fernández ${ }^{1}$ \\ Universidad Pablo de Olavide \\ Miguel Baldomero Ramírez-Fernández ${ }^{2}$ \\ Universidad Pablo de Olavide \\ Eva Ordóñez Olmedo ${ }^{3}$ \\ Universidad Pablo de Olavide
}

Resumen: En este trabajo se realiza una revisión del concepto de competencia a nivel académico y en toda la legislación vigente española respecto al currículo de la Educación Secundaria. También se contemplan los requisitos competenciales de los futuros profesores en su formación inicial y se recoge un diagnóstico del grado de adquisición secuencial a través de una síntesis de indicadores competenciales basados en los requeridos para este tipo de docencia. De esta manera, en un intento de evaluar el nivel competencial del alumnado que cursa el Máster Universitario que habilita para el ejercicio de la docencia como Profesor/a de Educación Secundaria, la Universidad Pablo de Olavide (en adelante UPO) de Sevilla ${ }^{4}$ ha recogido una propuesta de valoración de competencias de los futuros docentes durante el curso académico 2014-2015 mediante 21 indicadores competenciales clasificados en 5 secciones claves. En base a los resultados obtenidos, se deberían tomar medidas preventivas y los ajustes necesarios en aquellos indicadores competenciales que no han sido adquiridos progresivamente en los alumnos durante el periodo académico de estudio, analizar las causas de aquellos otros que no han sido superados por el $60 \%$ de los alumnos del Máster Universitario, y realizar una revisión del perfil de los estudiantes en base al bajo porcentaje de alumnos que adquirieron estos indicadores antes de comenzar el programa (por debajo del $30 \%$ del alumnado).

Palabras clave: Competencia, Evaluación, Calidad, Formación del Profesorado, Indicadores Competenciales.

Abstract: This paper makes a review of the concept of competence in academia and across the Spanish legislation regarding the curriculum of secondary education. It also includes the competency requirements for prospective teachers in their initial training and an assessment of the degree of sequence acquisition through a synthesis of competence indicators required for this type of teaching. Thus, in an attempt to assess the competence level of students enrolled in the Master's Degree entitling the holder to carry out teaching as Professor of Secondary Education, the University of Pablo de Olavide (hereafter UPO) in Seville has collected a proposal assessment of competence of future teachers during the academic year 2014-2015 by 21 competence indicators classified into 5 key sections. Based on the results obtained, should take preventive measures and adjustments required in those competence indicators that have not been acquired progressively students during the academic year of study, analyze the causes of those that

${ }^{1}$ Profesor Titular de Universidad de Didáctica y Organización Educativa de la UPO. Exdecano de la Facultad de Ciencias Sociales. Director del Máster de Formación del Profesorado (MAES) y del Máster de Educación para el Desarrollo. Director del grupo de investigación GEDUPO. Experto en Organización Educativa.

${ }^{2}$ Doctor en Educación y Ciencias Sociales con reconocimiento de Premio Extraordinario. Profesor Asociado LOU en el Área de Didáctica y Organización Escolar del Departamento de Educación y Psicología Social de la UPO. Inspector de Educación en la Delegación Territorial de Sevilla de la Consejería de Educación.

${ }^{3}$ Doctoranda en la línea de investigación de educación. Máster Universitario en Educación para el Desarrollo, Sensibilización Social y Cultura de Paz. Licenciada en Psicopedagogía. Graduada en Magisterio de Educación Primaria. Asesora en materia de competencias en el Centro de Estudios de Postgrado de la UPO.

4 http://www.upo.es/postgrado/Master-Oficial-Profesorado-de-Educacion-Secundaria-Obligatoria-yBachillerato-Formacion-Profesional-y-Ensenanza-de-Idiomas 
have not been surpassed by $60 \%$ of students in the Master, and to review the profile of the students based on the low percentage of them who acquired these indicators before starting the program (below $30 \%$ of students).

Keywords: Competence, Assessment, Quality, Teacher Training, Competence Indicators.

\section{Introducción}

Se va a introducir este artículo definiendo el concepto de competencia con el que se va a trabajar. Así, si se acude a la literatura científica al respecto, casi se puede afirmar que cada autor/a que ha abordado el tema de las competencias ha acuñado una definición propia. Más que proceder a una relación exhaustiva de diferentes definiciones se va a proceder a un pequeño análisis de las más relevantes, en aras de conseguir consensuar una definición que resulte operativa, para poder realizar el trabajo de síntesis de indicadores competenciales que se han diseñado en la UPO y que debe alcanzar nuestro alumnado universitario del Máster Universitario.

Dentro de las reformas que han abordado los países de la Unión Europea para incorporarse al Espacio Europeo de Educación Superior (en adelante EEES), uno de los estudios que más impacto ha generado en la adopción del modelo de enseñanza por competencias ha sido el proyecto Tuning ${ }^{5}$. En este proyecto se desarrollan algunas conclusiones acerca del aprendizaje por competencias:

"Por resultados del aprendizaje queremos significar el conjunto de competencias que incluye conocimientos, comprensión y habilidades que se espera que el estudiante domine, comprenda y demuestre después de completar un proceso corto o largo de aprendizaje...... Las competencias se pueden dividir en dos tipos: competencias genéricas, que en principio son independientes del área de estudio y competencias específicas para cada área temática."

En esta vasta investigación, que está ampliando sus efectos y resultados al entorno de América Latina, y sigue profundizando en sus conclusiones, se desarrolla la clasificación de las competencias genéricas:

"Competencias Instrumentales (habilidades cognoscitivas, capacidades metodológicas, destrezas tecnológicas o lingüísticas).

Competencias interpersonales (capacidades individuales, destrezas sociales).

Competencias sistémicas (comprensión, sensibilidad, conocimiento de sistemas; necesita la adquisición previa de las anteriores)"

Dentro de los procesos de reforma de los estudios universitarios en España, se procedió como primer paso en todas las titulaciones a la elaboración de una serie de "Libros Blancos" sobre cada una de ellas. Estos trabajos, auspiciados por la Agencia Nacional de Evaluación de la Calidad y Acreditación (en adelante ANECA) y elaborados desde una amplia red de universidades, ofrecían en cada caso una definición de competencia. Se recoge la proporcionada por el "Libro Blanco: Título de Grado en Pedagogía y Educación Social" ${ }^{\prime 6}$ como:

\footnotetext{
${ }^{5} \mathrm{http}: / /$ www.unideusto.org/tuningeu/

6 http://www.aneca.es/media/150392/libroblanco_pedagogia1_0305.pdf
} 
"Así, se definieron las competencias como el conjunto de conocimientos, habilidades, actitudes aplicadas en el desempeño de una profesión. Implica el ser, el saber, en sus distintas aplicaciones, y el saber hacer" (Agencia Nacional de Evaluación de la Calidad y Acreditación [ANECA], 2004, p. 155).

Estas definiciones surgen desde un foco de interés netamente académico y elaboran el constructo de competencia partiendo de elementos conocidos en la docencia universitaria. Así la definición de competencia aparece como un constructo complejo en el que el desarrollo personal del alumnado se enfatiza por encima de la simple asimilación de contenidos. Los aprendizajes adquieren un carácter eminentemente aplicativo, que se relaciona con visiones globales y cambiantes de la realidad. De este modo, es evidente que se rompe la visión disciplinar y los aprendizajes deben realizarse desde entornos interdisciplinares con fuertes componentes de colaboración entre docentes de diferentes áreas de conocimiento. El concepto de competencia es global, holístico e integra elementos cognitivos, afectivos y comportamentales. No es de extrañar que, abordado desde este punto vista, el concepto de competencia pueda parecer como algo complejo de abordar desde la planificación de la enseñanza, no sólo universitaria. Y que haya generado, incluso, determinadas corrientes de pensamiento y trabajo científico y académico críticas con este enfoque.

Desde el punto de vista académico y pedagógico se ha realizado un enorme esfuerzo desde distintos ámbitos por hacer más comprensible y manejable el concepto de competencia en la planificación, desarrollo y evaluación de procesos de enseñanza universitaria.

Así uno de los pasos con los que se suele iniciar esta aclaración es situando adecuadamente los términos que suelen, históricamente, vincularse con el término de competencia. Un trabajo interesante en esta línea es el recogido por la Agencia para la Calidad del Sistema Universitario de Cataluña, que ha publicado una serie de Guías para la planificación y evaluación de competencias. De entre ellas, se destaca la publicada sobre el área de Ciencias Sociales", que en su punto "Aclarando Conceptos", ofrece:

"Los rasgos y las características personales son los cimientos del aprendizaje, la base innata desde la que se pueden construir las experiencias subsiguientes. Las diferencias en rasgos y características ayudan a explicar por qué las personas escogen diferentes experiencias de aprendizaje y adquieren diferentes niveles y tipologías de conocimientos y habilidades. Los conocimientos, las habilidades y las actitudes se desarrollan a partir de las experiencias de aprendizaje, que, si se definen de una manera amplia, incluyen tanto la escuela como el trabajo, la familia, la participación social, etc.

Las competencias son combinaciones de conocimientos, habilidades y actitudes adquiridas. Se desarrollan a partir de experiencias de aprendizaje integrativas en las que los conocimientos y las habilidades interactúan con el fin de dar una respuesta eficiente en la tarea que se ejecuta. Las demostraciones comportan la aplicación de las competencias aprendidas, en contextos específicos." (Agència per a la Qualitat del Sistema Universitari de Catalunya [AQU], 2009, p. 17-18).

\footnotetext{
${ }^{7}$ http://www.aqu.cat/publicacions/guies_competencies/guia_ciencies_socials_es.html
} 
Dado que las competencias son el resultado de combinar conocimientos y habilidades, parece que en procesos educativos de larga duración, como el correspondiente a las enseñanzas universitarias, el logro de las competencias se conseguirá hacia el final del proceso. Así parece útil diferenciar la competencia de otros conceptos vinculados con la programación didáctica y la evaluación, para facilitar la coordinación entre el profesorado que participe en un mismo título, como se muestra en la Tabla 1.

\begin{tabular}{l|l}
\hline \multicolumn{1}{c|}{ Conceptos } & $\begin{array}{l}\text { Competencias que los estudiantes del Máster Universitario } \\
\text { deben adquirir para la obtención del Título }\end{array}$ \\
\hline Objetivos & $\begin{array}{l}\text { Son afirmaciones relativas a la docencia, redactadas desde el } \\
\text { punto de vista de aquello que intentará cubrir el profesorado } \\
\text { con un determinado bloque de aprendizaje (módulo, materia, } \\
\text { asignatura, etc.). Están escritos desde el punto de vista del } \\
\text { profesorado. Pueden incluir conocimientos y habilidades de } \\
\text { manera aislada. }\end{array}$ \\
\hline Resultados & $\begin{array}{l}\text { Son afirmaciones sobre las que se espera que un estudiante } \\
\text { pueda conocer, comprender y ser capaz de demostrar después } \\
\text { aprendizaje haber completado un proceso de aprendizaje (módulo, } \\
\text { asignatura, materia, curso, etc.). Se basan en lo que el } \\
\text { estudiante ha alcanzado en vez de en cuáles son las } \\
\text { intenciones del profesorado. Se centran en aquello que puede } \\
\text { demostrar el estudiante al finalizar la actividad de aprendizaje. } \\
\text { Pueden incluir conocimientos y habilidades aisladamente. De } \\
\text { la misma manera que los objetivos, se pueden describir al } \\
\text { finalizar cualquier unidad (módulo, asignatura, etc.). }\end{array}$ \\
\hline Competencias & $\begin{array}{l}\text { Implican el uso integrado de conocimientos, habilidades y } \\
\text { actitudes en la acción. Por su naturaleza, sólo se podrán } \\
\text { alcanzar estadios finales del proceso educativo (practicum, } \\
\text { trabajos final de carrera, etc.). }\end{array}$ \\
\hline
\end{tabular}

Tabla 1. Diferenciación de competencia de otros términos vinculados con el diseño curricular. Fuente: Agència per a la Qualitat del Sistema Universitari de Catalunya [AQU] (2009).

De este modo se concluye la siguiente definición de competencia:

"La competencia es la combinación de habilidades, actitudes y conocimientos necesarios para desarrollar una tarea de manera eficaz. Las competencias se demuestran en la acción y, por lo tanto, sólo son evaluables si hay actividades de aplicación. Las competencias son aprendidas y se desarrollan a partir de actividades que permiten integrar habilidades, actitudes y conocimientos aprendidos anteriormente y quizás de manera separada.

La formulación de la competencia requiere de los elementos siguientes:

- Un verbo activo que identifique una acción que genere un resultado visualizable. De esta manera, hay que evitar el uso de verbos como conocer o comprender y utilizar otras formas verbales como describe, identifica, reconoce, clasifica, compara, evalúa o valora, formula, argumenta, calcula, planifica, diseña, etc.

- La descripción del objeto de la acción y el contexto en que se aplica. La competencia tiene que hacer referencia al campo disciplinario en el que se fundamenta." (Agència per a la Qualitat del Sistema Universitari de Catalunya [AQU], 2009, p. 19). 
Por medio de estos trabajos y estudios se puede apreciar que, desde el punto de vista académico, se pueden ir aclarando las tareas docentes que hay que emprender en un proceso de enseñanza universitaria con las competencias como eje de desarrollo. Al igual que con la propia definición de competencia, todos los autores que se han aproximado a la conceptualización de este concepto han aportado sus elementos. En este sentido, se puede concluir que todos están de acuerdo, al menos, en tres:

- Saber: El conjunto de conocimientos que permiten alcanzar la competencia.

- Saber hacer: Conjunto de técnicas, herramientas, procedimientos, en definitiva, habilidades que es necesario aplicar para alcanzar la competencia.

- Saber estar: Actitudes ajustadas a normas, reglas, valores o paradigmas sociales, científicos o personales que suponen conseguir la competencia.

Estos elementos de la competencia son ampliados por diferentes autores a varios aspectos que pueden vincularse o englobarse en los anteriores. Estos otros elementos pueden ser, querer hacer y poder hacer; saber a saber y saber desaprender o saber transferir (Domínguez y Hermosilla, 2010).

\section{Metodología}

Para la elaboración del presente artículo se realizó la revisión exhaustiva de toda la normativa vigente española relacionada con la formación profesional inicial de los futuros aspirantes a Profesores de Educación Secundaria a fecha de julio de 2015. De este modo, se ha secuenciado su búsqueda en las competencias que deben enseñar los futuros docentes y las competencias que deben superar para enseñar dichos profesores en estas etapas educativas. Posteriormente, se ha sintetizado en un estudio de caso para los estudiantes (aspirantes a Profesores de Educación Secundaria) del Máster Universitario de la UPO una serie de indicadores competenciales y su grado de adquisición sobre los mismos durante el curso académico 2014-2015.

\subsection{Las competencias en el currículo básico de la Educación Secundaria}

En el Real Decreto $1105 / 2014^{8}$, la Ley Orgánica $8 / 2013^{9}$ modificó el artículo 6 de la Ley Orgánica $2 / 2006^{10}$, para definir el currículo como la regulación de los elementos que determinan los procesos de enseñanza y aprendizaje para cada una de las enseñanzas. El currículo estará integrado por los objetivos de cada enseñanza y etapa educativa; las competencias, o capacidades para activar y aplicar de forma integrada los contenidos propios de cada enseñanza y etapa educativa, para lograr la realización adecuada de actividades y la resolución eficaz de problemas complejos; los contenidos, o conjuntos de conocimientos, habilidades, destrezas y actitudes que contribuyen al logro de los objetivos de cada enseñanza y etapa educativa y a la adquisición de competencias; la metodología didáctica, que comprende tanto la descripción de las prácticas docentes como la organización del trabajo de los docentes; los estándares y resultados de aprendizaje evaluables; y los criterios de evaluación del grado de adquisición de las competencias y del logro de los objetivos de cada enseñanza y etapa educativa. Los contenidos se ordenan en asignaturas, que se clasifican en materias, ámbitos, áreas y módulos en función de las enseñanzas, las etapas educativas o los programas en que participe el alumnado.

\footnotetext{
${ }^{8}$ Real Decreto 1105/2014, de 26 de diciembre, por el que se establece el currículo básico de la Educación Secundaria Obligatoria y del Bachillerato.

${ }^{9}$ Ley Orgánica 8/2013, de 9 de diciembre, para la Mejora de la Calidad Educativa.

${ }^{10}$ Ley Orgánica 2/2006, de 3 de mayo, de Educación.
} 
En este sentido, en línea con la Recomendación 2006/962/CE ${ }^{11}$, este Real Decreto se basa en la potenciación del aprendizaje por competencias, integradas en los elementos curriculares para propiciar una renovación en la práctica docente y en el proceso de enseñanza y aprendizaje. Se proponen nuevos enfoques en el aprendizaje y evaluación, que han de suponer un importante cambio en las tareas que han de resolver los alumnos y planteamientos metodológicos innovadores. La competencia supone una combinación de habilidades prácticas, conocimientos, motivación, valores éticos, actitudes, emociones, y otros componentes sociales y de comportamiento que se movilizan conjuntamente para lograr una acción eficaz. Se contemplan, pues, como conocimiento en la práctica, un conocimiento adquirido a través de la participación activa en prácticas sociales que, como tales, se pueden desarrollar tanto en el contexto educativo formal, a través del currículo, como en los contextos educativos no formales e informales.

Las competencias, por tanto, se conceptualizan como un «saber hacer» que se aplica a una diversidad de contextos académicos, sociales y profesionales. Para que la transferencia a distintos contextos sea posible resulta indispensable una comprensión del conocimiento presente en las competencias, y la vinculación de éste con las habilidades prácticas o destrezas que las integran.

El aprendizaje por competencias favorece los propios procesos de aprendizaje y la motivación por aprender, debido a la fuerte interrelación entre sus componentes: el concepto se aprende de forma conjunta al procedimiento de aprender dicho concepto.

Se adopta la denominación de las competencias clave definidas por la Unión Europea. Se considera que "las competencias clave son aquellas que todas las personas precisan para su realización y desarrollo personal, así como para la ciudadanía activa, la inclusión social y el empleo". Se identifican siete competencias clave esenciales para el bienestar de las sociedades europeas, el crecimiento económico y la innovación, y se describen los conocimientos, las capacidades y las actitudes esenciales vinculadas a cada una de ellas.

El rol del docente es fundamental, pues debe ser capaz de diseñar tareas o situaciones de aprendizaje que posibiliten la resolución de problemas, la aplicación de los conocimientos aprendidos y la promoción de la actividad de los estudiantes.

La revisión curricular tiene muy en cuenta las nuevas necesidades de aprendizaje. El aprendizaje basado en competencias se caracteriza por su transversalidad, su dinamismo y su carácter integral. El proceso de enseñanza-aprendizaje competencial debe abordarse desde todas las áreas de conocimiento, y por parte de las diversas instancias que conforman la comunidad educativa, tanto en los ámbitos formales como en los no formales e informales; su dinamismo se refleja en que las competencias no se adquieren en un determinado momento y permanecen inalterables, sino que implican un proceso de desarrollo mediante el cual los individuos van adquiriendo mayores niveles de desempeño en el uso de las mismas.

Para lograr este proceso de cambio curricular es preciso favorecer una visión interdisciplinar y, de manera especial, posibilitar una mayor autonomía a la función docente, de forma que permita satisfacer las exigencias de una mayor personalización de la educación, teniendo en cuenta el principio de especialización del profesorado.

El currículo básico de las asignaturas correspondientes a la Educación Secundaria Obligatoria y el Bachillerato se ha diseñado partiendo de los objetivos propios de la etapa y de las competencias que se van a desarrollar a lo largo de la misma, mediante el establecimiento de bloques de contenidos en las asignaturas troncales, y criterios de evaluación y estándares de aprendizaje evaluables en todas las asignaturas, que serán

\footnotetext{
${ }^{11}$ Recomendación 2006/962/CE del Parlamento Europeo y del Consejo, de 18 de diciembre de 2006, sobre las competencias clave para el aprendizaje permanente.
} 
referentes en la planificación de la concreción curricular y en la programación didáctica.

Así pues, en el artículo 2.1.c del Real Decreto citado se define el elemento curricular competencias como las "capacidades para aplicar de forma integrada los contenidos propios de cada enseñanza y etapa educativa, con el fin de lograr la realización adecuada de actividades y la resolución eficaz de problemas complejos".

\subsection{Relaciones entre las competencias, los contenidos y los criterios de evaluación en la Educación Secundaria}

En la Orden ECD/65/2015 ${ }^{12}$ se relacionan las siguientes competencias clave del currículo, como se muestra en la Tabla 2.

\begin{tabular}{c}
\hline Competencias clave en el currículo de Educación Secundaria \\
\hline Comunicación lingüística \\
\hline Competencia matemática y competencias básicas en ciencia y \\
tecnología \\
\hline Competencia digital \\
\hline Aprender a aprender \\
\hline Competencias sociales y cívicas \\
\hline Sentido de iniciativa y espíritu emprendedor \\
Conciencia y expresiones culturales \\
\hline Tabla 2. Clasificación de las competencias clave en el currículo de \\
Educación Secundaria. \\
Fuente: Ministerio de Educación, Cultura y Deporte [MECD] (2015).
\end{tabular}

En el artículo 4 de la Orden se establece la estrecha vinculación de las competencias clave a los objetivos definidos para la Educación Secundaria. Para ello se hace necesario diseñar estrategias para promover y evaluar las competencias desde las etapas educativas iniciales e intermedias hasta su posterior consolidación en etapas superiores, que llevarán a los alumnos a desarrollar actitudes y valores, así como un conocimiento de base conceptual y un uso de técnicas y estrategias que favorecerán su incorporación a la vida adulta y que servirán de cimiento para su aprendizaje a lo largo de su vida.

De esta manera, la adquisición eficaz de las competencias clave por parte del alumnado y su contribución al logro de los objetivos de las etapas educativas, desde un carácter interdisciplinar y transversal, requiere del diseño de actividades de aprendizaje integradas que permitan avanzar hacia los resultados de aprendizaje de más de una competencia al mismo tiempo.

En el artículo 5 de la Orden se recoge que las competencias clave deben estar integradas en las áreas o materias de las propuestas curriculares, y en ellas definirse, explicitarse y desarrollarse suficientemente los resultados de aprendizaje que los alumnos deben conseguir. Deben desarrollarse en los ámbitos de la educación formal, no formal e informal a lo largo de las etapas educativas, y en la educación permanente a lo largo de toda la vida.

Todas las áreas o materias del currículo deben participar, desde su ámbito correspondiente, en el desarrollo de las distintas competencias del alumnado. Por tanto, la selección de los contenidos y las metodologías debe asegurar el desarrollo de las competencias clave a lo largo de la vida académica.

\footnotetext{
12 Orden ECD/65/2015, de 21 de enero, por la que se describen las relaciones entre las competencias, los contenidos y los criterios de evaluación de la Educación Primaria, la educación secundaria obligatoria y el bachillerato.
} 
En base a lo anterior, los criterios de evaluación deben servir de referencia para valorar lo que el alumnado sabe y sabe hacer en cada área o materia. Se desglosan en estándares de aprendizaje evaluables. Para valorar el desarrollo competencial del alumnado, serán estos estándares de aprendizaje evaluables, como elementos de mayor concreción, observables y medibles, los que, al ponerse en relación con las competencias clave, permitirán graduar el rendimiento o desempeño alcanzado en cada una de ellas.

Así pues, el conjunto de estándares de aprendizaje evaluables de un área o materia determinada dará lugar a su perfil de área o materia. Dado que los estándares de aprendizaje evaluables se ponen en relación con las competencias, este perfil permitirá identificar aquellas competencias que se desarrollan a través de esa área o materia.

Por último, como todas las áreas y materias deben contribuir al desarrollo competencial, el conjunto de estándares de aprendizaje evaluables de las diferentes áreas o materias que se relacionan con una misma competencia da lugar al perfil de esa competencia (perfil de competencia). La elaboración de este perfil facilitará la evaluación competencial del alumnado.

\subsubsection{Estrategias metodológicas para trabajar por competencias en el aula}

En el artículo 6 de la Orden se mencionan las siguientes estrategias metodológicas para trabajar por competencias en el aula, como se recogen en la Tabla 3.

\section{Estrategias metodológicas}

1. Los métodos deben partir de la perspectiva del docente como orientador, promotor y facilitador del desarrollo competencial en el alumnado; además, deben enfocarse a la realización de tareas o situaciones-problema, planteadas con un objetivo concreto, que el alumnado debe resolver haciendo un uso adecuado de los distintos tipos de conocimientos, destrezas, actitudes y valores; asimismo, deben tener en cuenta la atención a la diversidad y el respeto por los distintos ritmos y estilos de aprendizaje mediante prácticas de trabajo individual y cooperativo.

2. Cualquiera de las metodologías seleccionadas por los docentes para favorecer el desarrollo competencial de los alumnos debe ajustarse al nivel competencial inicial de estos. Se hace necesario secuenciar la enseñanza de tal modo que se parta de aprendizajes más simples para avanzar gradualmente hacia otros más complejos.

3. Despertar y mantener la motivación hacia el aprendizaje en el alumnado, lo que implica un nuevo planteamiento del papel del alumno, activo y autónomo, consciente de ser el responsable de su aprendizaje.

4. Los métodos docentes deberán favorecer la motivación por aprender en los alumnos y, a tal fin, los profesores han de ser capaces de generar en ellos la curiosidad y la necesidad por adquirir los conocimientos, las destrezas y las actitudes y valores presentes en las competencias. Asimismo, es necesario que los profesores procuren todo tipo de ayudas para que los estudiantes comprendan lo que aprenden, sepan para qué lo aprenden y sean capaces de usar lo aprendido en distintos contextos dentro y fuera del aula.

5. Usar metodologías activas y contextualizadas que faciliten la participación e implicación del alumnado y la adquisición y uso de conocimientos en situaciones reales harán que se generen aprendizajes más transferibles y duraderos. Las metodologías activas han de apoyarse en estructuras de aprendizaje cooperativo, de forma que, a través de la resolución conjunta de las tareas, los miembros del grupo conozcan las estrategias utilizadas por sus compañeros y puedan aplicarlas a situaciones similares. 
6. Usar estrategias interactivas que permitan compartir y construir el conocimiento y dinamizar la sesión de clase mediante el intercambio verbal y colectivo de ideas. Las metodologías que contextualizan el aprendizaje y permiten el aprendizaje por proyectos, los centros de interés, el estudio de casos o el aprendizaje basado en problemas favorecen la participación activa, la experimentación y un aprendizaje funcional que va a facilitar el desarrollo de las competencias, así como la motivación de los alumnos al contribuir decisivamente a la transferibilidad de los aprendizajes.

7. El trabajo por proyectos se basa en la propuesta de un plan de acción con el que se busca conseguir un determinado resultado práctico. Esta metodología pretende ayudar al alumnado a organizar su pensamiento favoreciendo en ellos la reflexión, la crítica, la elaboración de hipótesis y la tarea investigadora a través de un proceso en el que cada uno asume la responsabilidad de su aprendizaje, aplicando sus conocimientos y habilidades a proyectos reales.

8. Usar el portfolio aporta información extensa sobre el aprendizaje del alumnado, refuerza la evaluación continua y permite compartir resultados de aprendizaje. Además, potencia su autonomía y desarrolla su pensamiento crítico y reflexivo.

9. La implicación del profesorado en la selección y uso de materiales y recursos didácticos constituye un aspecto esencial de la metodología. Además, deberán ser adaptados a los distintos niveles y a los diferentes estilos y ritmos de aprendizaje de los alumnos, con el objeto de atender a la diversidad en el aula y personalizar los procesos de construcción de los aprendizajes. Se debe potenciar el uso de una variedad de materiales y recursos, considerando especialmente la integración de las Tecnologías de la Información y la Comunicación (en adelante TIC) en el proceso de enseñanzaaprendizaje que permiten el acceso a recursos virtuales.

10. Se hace necesaria una adecuada coordinación entre los docentes sobre las estrategias metodológicas y didácticas que se utilicen. Los equipos educativos deben plantearse una reflexión común y compartida sobre la eficacia de las diferentes propuestas metodológicas con criterios comunes y consensuados. Esta coordinación y la existencia de estrategias conexionadas permiten abordar con rigor el tratamiento integrado de las competencias y progresar hacia una construcción colaborativa del conocimiento.

Tabla 3. Estrategias metodológicas para trabajar por competencias en el aula en el currículo de Educación Secundaria.

Fuente: Ministerio de Educación, Cultura y Deporte [MECD] (2015).

\subsubsection{La evaluación de las competencias clave}

El artículo 7 de la Orden establece los siguientes aspectos en cuanto a la evaluación de las competencias clave, como se indica en la Tabla 4.

\begin{tabular}{l} 
Evaluación de competencias clave \\
\hline 1. Se hace necesario elegir, siempre que sea posible, estrategias e instrumentos para \\
evaluar al alumnado de acuerdo con sus desempeños en la resolución de problemas \\
que simulen contextos reales, movilizando sus conocimientos, destrezas, valores y \\
actitudes. \\
\hline 2. Han de establecerse las relaciones de los estándares de aprendizaje evaluables \\
con las competencias a las que contribuyen, para lograr la evaluación de los niveles \\
de desempeño competenciales alcanzados por el alumnado. \\
\hline 3. La evaluación del grado de adquisición de las competencias debe estar integrada \\
con la evaluación de los contenidos, en la medida en que ser competente supone \\
movilizar los conocimientos, destrezas, actitudes y valores para dar respuesta a las \\
situaciones planteadas, dotar de funcionalidad a los aprendizajes y aplicar lo que se \\
aprende desde un planteamiento integrador. \\
\hline 4. Los niveles de desempeño de las competencias se podrán medir a través de \\
\hline
\end{tabular}


indicadores de logro, tales como rúbricas o escalas de evaluación. Estos indicadores de logro deben incluir rangos dirigidos a la evaluación de desempeños, que tengan en cuenta el principio de atención a la diversidad.

5. El profesorado establecerá las medidas que sean necesarias para garantizar que la evaluación del grado de dominio de las competencias del alumnado con discapacidad se realice de acuerdo con los principios de no discriminación y accesibilidad y diseño universal.

6. El profesorado debe utilizar procedimientos de evaluación variados y como una herramienta esencial para mejorar la calidad de la educación. Asimismo, es necesario incorporar estrategias que permitan la participación del alumnado en la evaluación de sus logros, como la autoevaluación, la evaluación entre iguales o la coevaluación. En todo caso, los distintos procedimientos de evaluación utilizables, como la observación sistemática del trabajo de los alumnos, las pruebas orales y escritas, el portfolio, los protocolos de registro, o los trabajos de clase, permitirán la integración de todas las competencias en un marco de evaluación coherente.

7. Las evaluaciones externas de fin de etapa previstas en la Ley Orgánica 8/2013 tendrán en cuenta, tanto en su diseño como en su evaluación los estándares de aprendizaje evaluable del currículo.

Tabla 4. Sugerencias de evaluación de competencias clave en el currículo de Educación Secundaria.

Fuente: Ministerio de Educación, Cultura y Deporte [MECD] (2015).

\subsection{La formación inicial del profesorado de secundaria}

La Ley Orgánica 2/2006 se refiere en el capítulo III de su título III a la formación del profesorado, tanto inicial como permanente. Respecto de la primera dispone que se ajustará a las necesidades de titulación y de cualificación requeridas por la ordenación general del sistema educativo.

Asimismo, destaca la relevancia de la experiencia profesional a la hora de facilitar y tutorizar la incorporación a la docencia del profesorado y el acceso a la formación permanente como un derecho y una obligación de todo el personal docente y una responsabilidad de las Administraciones educativas y de los propios centros.

En cuanto a la Comunidad Autónoma de Andalucía (España), la Ley 17/2007 ${ }^{13}$ dedica el capítulo II de su título I al profesorado, ocupándose en la sección $3 .^{\mathrm{a}}$ de dicho capítulo de la formación inicial y permanente del profesorado y del Sistema Andaluz de Formación Permanente del Profesorado. Además, en el artículo 18 dispone que el componente esencial de la formación inicial será la relación permanente e interactiva entre la teoría y la práctica y la preparación para la dirección de los procesos de enseñanza y aprendizaje y de desarrollo personal del alumnado.

En este sentido, el Decreto 93/2013 ${ }^{14}$ recoge que el aumento de la calidad de la formación debe constituir una de las metas prioritarias para garantizar una docencia de alto nivel, ofrecer una formación inicial del profesorado adecuada, así como un desarrollo profesional permanente del personal docente y asesor que, basado en la autoevaluación y en el desarrollo de sus competencias profesionales, contribuya a la calidad del sistema educativo y ofrezca resultados claros y visibles de mejora en los rendimientos del alumnado.

En el citado decreto se establece que la formación inicial del profesorado se ajustará a las necesidades de titulación y de cualificación requeridas por la ordenación general del sistema educativo y se regulará según lo recogido en la Ley Orgánica 2/2006 y en la correspondiente normativa de desarrollo. Su contenido garantizará las

\footnotetext{
${ }^{13}$ Ley 17/2007, de 10 de diciembre, de Educación de Andalucía.

14 Decreto 93/2013, de 27 de agosto, por el que se regula la formación inicial y permanente del profesorado en la Comunidad Autónoma de Andalucía, así como el Sistema Andaluz de Formación Permanente del Profesorado.
} 
competencias profesionales adecuadas para afrontar los retos del sistema educativo y adaptar las enseñanzas a las nuevas necesidades formativas del alumnado.

Atendiendo al artículo 4 del decreto, de entre las competencias profesionales que permitirá desarrollar la formación inicial del profesorado se encuentra la de "utilizar estrategias metodológicas y recursos didácticos que propicien el desarrollo integrado de las competencias básicas en el alumnado, entre los que se encuentran el trabajo por tareas y proyectos".

En el artículo 5 se hace referencia a los planes de estudios y la fase de prácticas. De esta manera, la Consejería competente en materia de educación promoverá que los planes de estudios de grado y máster relativos a la formación inicial del profesorado realizados por las universidades contemplen el máximo número de créditos establecido reglamentariamente para la fase de prácticas, con objeto de garantizar la participación del alumnado en todas las actividades que se llevan a cabo en un centro docente, incluidas la docencia directa, reuniones de órganos de coordinación docente, planificación y programación de las actividades docentes y complementarias y funciones de tutoría con el alumnado y sus padres, madres o quienes ejerzan su tutela.

La fase de prácticas deberá proporcionar al futuro profesorado una adecuada competencia profesional, dotándole del conocimiento de sus derechos, funciones y deberes, así como de los distintos aspectos del trabajo en un centro docente con especial énfasis en la importancia del trabajo en equipo, en el sentido de pertenencia a una organización y en la necesidad de contribuir a la mejora continuada de la misma, de acuerdo con lo que a tales efectos se establezca por Orden de la persona titular de la Consejería competente en materia de educación. En este sentido, las actividades formativas se enfocarán desde una perspectiva práctica, combinando ideas y experiencias para proporcionar estrategias metodológicas, materiales y recursos en un contexto educativo real.

El artículo 9 establece que la organización, realización y evaluación de la fase de prácticas para el ingreso en los cuerpos docentes se llevará a cabo de conformidad con lo establecido en la sección primera del capítulo II del Decreto 302/2010

\subsection{Habilitación para el ejercicio de la docencia al profesorado de secundaria}

En España, el Máster Universitario, que habilita para el ejercicio de la docencia como Profesor/a de Educación Secundaria Obligatoria, Bachillerato, Formación Profesional y Enseñanza de Idiomas, es requisito específico para el ingreso en los Cuerpos de Profesores de Enseñanza Secundaria, Profesores Técnicos de Formación Profesional, Profesores de Música y Artes Escénicas, Profesores de Artes Plásticas y Diseño, maestros de Taller de Artes Plásticas y Diseño y Profesores de Escuelas Oficiales de Idiomas, según se establece en el Real Decreto 276/2007 ${ }^{16}$.

Para el ingreso en el Máster se establece como requisito de acceso la acreditación del dominio de las competencias relativas a la especialización que se desee cursar, mediante la realización de una prueba diseñada al efecto por las Universidades, de la que quedarán exentos quienes estén en posesión de alguna de las titulaciones universitarias que se correspondan con la especialización elegida. Además, los candidatos deberán certificar el nivel B1 del Marco Común Europeo de Referencia para las Lenguas en un idioma moderno. Los estudiantes extranjeros deberán certificar el nivel B1 en lengua española.

\footnotetext{
${ }^{15}$ Decreto 302/2010, de 1 de julio, por el que se ordena la función pública docente y se regula la selección del profesorado y la provisión de puestos de trabajo docentes.

${ }^{16}$ Real Decreto 276/2007, de 23 de febrero, por el que se aprueba el Reglamento de ingreso, accesos y adquisición de nuevas especialidades en los cuerpos docentes a que se refiere la Ley Orgánica 2/2006, de 3 de mayo, de Educación, y se regula el régimen transitorio de ingreso a que se refiere la disposición transitoria decimoséptima de la citada ley.
} 
En la Orden ECI/3858/2007 ${ }^{17}$ se establecen los requisitos respecto a determinados apartados del anexo I del Real Decreto $1393 / 2007^{18}$ relativo a la memoria para la solicitud de verificación de títulos oficiales. En la Tabla 5 se muestran las competencias que los estudiantes aspirantes a Profesores de Educación Secundaria deben adquirir.

Competencias que los estudiantes del Máster Universitario deben adquirir para
la obtención del Título

2. Planificar, desarrollar y evaluar el proceso de enseñanza y aprendizaje potenciando procesos educativos que faciliten la adquisición de las competencias propias de las respectivas enseñanzas, atendiendo al nivel y formación previa de los estudiantes así como la orientación de los mismos, tanto individualmente como en colaboración con otros docentes y profesionales del centro.

3. Buscar, obtener, procesar y comunicar información (oral, impresa, audiovisual, digital o multimedia), transformarla en conocimiento y aplicarla en los procesos de enseñanza y aprendizaje en las materias propias de la especialización cursada.

4. Concretar el currículo que se vaya a implantar en un centro docente participando en la planificación colectiva del mismo; desarrollar y aplicar metodologías didácticas tanto grupales como personalizadas, adaptadas a la diversidad de los estudiantes.

5. Diseñar y desarrollar espacios de aprendizaje con especial atención a la equidad, la educación emocional y en valores, la igualdad de derechos y oportunidades entre hombres y mujeres, la formación ciudadana y el respeto de los derechos humanos que faciliten la vida en sociedad, la toma de decisiones y la construcción de un futuro sostenible.

6. Adquirir estrategias para estimular el esfuerzo del estudiante y promover su capacidad para aprender por sí mismo y con otros, y desarrollar habilidades de pensamiento y de decisión que faciliten la autonomía, la confianza e iniciativa personales.

7. Conocer los procesos de interacción y comunicación en el aula, dominar destrezas y habilidades sociales necesarias para fomentar el aprendizaje y la convivencia en el aula, y abordar problemas de disciplina y resolución de conflictos.

8. Diseñar y realizar actividades formales y no formales que contribuyan a hacer del centro un lugar de participación y cultura en el entorno donde esté ubicado; desarrollar las funciones de tutoría y de orientación de los estudiantes de manera colaborativa y coordinada; participar en la evaluación, investigación y la innovación de los procesos de enseñanza y aprendizaje.

9. Conocer la normativa y organización institucional del sistema educativo y modelos de mejora de la calidad con aplicación a los centros de enseñanza.

10. Conocer y analizar las características históricas de la profesión docente, su situación actual, perspectivas e interrelación con la realidad social de cada época.

11. Informar y asesorar a las familias acerca del proceso de enseñanza y aprendizaje y sobre la orientación personal, académica y profesional de sus hijos.

Tabla 5. Competencias que los estudiantes aspirantes a Profesores de Educación Secundaria deben adquirir para la obtención del Título del Máster. Fuente: Ministerio de Educación, Cultura y Deporte [MECD] (2007).

${ }^{17}$ Orden ECI/3858/2007, de 27 de diciembre, por la que se establecen los requisitos para la verificación de los títulos universitarios oficiales que habiliten para el ejercicio de las profesiones de Profesor de Educación Secundaria Obligatoria y Bachillerato, Formación Profesional y Enseñanzas de Idiomas.

${ }^{18}$ Real Decreto 1393/2007, de 29 de octubre, por el que se establece la ordenación de las enseñanzas universitarias oficiales, relativo a la memoria para la solicitud de verificación de títulos oficiales. 
Los planes de estudios del citado Máster universitario tendrán una duración de 60 créditos europeos y se estructurarán teniendo en cuenta las materias y ámbitos docentes en Educación Secundaria obligatoria y bachillerato, formación profesional, enseñanzas artísticas, enseñanzas de idiomas y enseñanzas deportivas. Con carácter general, han de ser presenciales, al menos, en el $80 \%$ de los créditos totales del Máster, incluido necesariamente el Practicum. Las Universidades que por su especificidad diseñan, programan y desarrollan las enseñanzas exclusivamente a distancia, han de garantizar que el Practicum tenga carácter presencial.

El Practicum se realizará en colaboración con las instituciones educativas establecidas mediante convenios entre Universidades y Administraciones Educativas. Las instituciones educativas participantes en la realización del Practicum habrán de estar reconocidas como centros de prácticas, así como los tutores encargados de la orientación y tutela de los estudiantes.

En la Tabla 6 se recogen las competencias que deberá adquirirse en cada uno de los módulos que componen el Máster Universitario.

\begin{tabular}{ll}
\hline Módulo & \multicolumn{1}{c}{$\begin{array}{c}\text { Competencias que los estudiantes del Máster Universitario } \\
\text { deben adquirir para cada módulo }\end{array}$} \\
\hline & Conocer las características de los estudiantes, sus contextos \\
& sociales y motivaciones. Comprender el desarrollo de la \\
personalidad de estos estudiantes y las posibles disfunciones que \\
afectan al aprendizaje. Elaborar propuestas basadas en la \\
adquisición de conocimientos, destrezas y aptitudes intelectuales \\
yemocionales. Identificar y planificar la resolución de situaciones \\
educativas que afectan a estudiantes con diferentes capacidades y \\
diferentes ritmos de aprendizaje.
\end{tabular}

Aprendizaje y desarrollo de la personalidad.

Procesos y contextos educativos.

Sociedad, familia y Educación.

(12 créditos europeos)
Conocer los procesos de interacción y comunicación en el aula y en el centro, abordar y resolver posibles problemas. Conocer la evolución histórica del sistema educativo en nuestro país. Conocer y aplicar recursos y estrategias de información, tutoría y orientación académica y profesional. Promover acciones de educación emocional, en valores y formación ciudadana. Participar en la definición del proyecto educativo y en las actividades generales del centro atendiendo a criterios de mejora de la calidad, atención a la diversidad, prevención de problemas de aprendizaje y convivencia.

Relacionar la educación con el medio y comprender la función educadora de la familia y la comunidad, tanto en la adquisición de competencias y aprendizajes como en la educación en el respeto de los derechos y libertades, en la igualdad de derechos y oportunidades entre hombres y mujeres y en la igualdad de trato y no discriminación de las personas con discapacidad. Conocer la evolución histórica de la familia, sus diferentes tipos y la incidencia del contexto familiar en la educación. Adquirir habilidades sociales en la relación y orientación familiar.

Específico Conocer el valor formativo y cultural de las materias correspondientes a la especialización y los contenidos que se cursan en las respectivas enseñanzas. Conocer la historia y los desarrollos recientes de las materias y sus perspectivas para poder transmitir una visión dinámica de las mismas. Conocer contextos 
Aprendizaje y enseñanza de las materias correspondientes.

Innovación docente e iniciación a la investigación educativa.

(24 créditos europeos) y situaciones en que se usan o aplican los diversos contenidos curriculares. En formación profesional, conocer la evolución del mundo laboral, la interacción entre sociedad, trabajo y calidad de vida, así como la necesidad de adquirir la formación adecuada para la adaptación a los cambios y transformaciones que puedan requerir las profesiones. En el caso de la orientación psicopedagógica y profesional, conocer los procesos y recursos para la prevención de problemas de aprendizaje y convivencia, los procesos de evaluación y de orientación académica y profesional.

Conocer los desarrollos teórico-prácticos de la enseñanza y el aprendizaje de las materias correspondientes. Transformar los currículos en programas de actividades y de trabajo. Adquirir criterios de selección y elaboración de materiales educativos. Fomentar un clima que facilite el aprendizaje y ponga en valor las aportaciones de los estudiantes. Integrar la formación en comunicación audiovisual y multimedia en el proceso de enseñanza-aprendizaje. Conocer estrategias y técnicas de evaluación y entender la evaluación como un instrumento de regulación y estímulo al esfuerzo.

Conocer y aplicar propuestas docentes innovadoras en el ámbito de la especialización cursada. Analizar críticamente el desempeño de la docencia, de las buenas prácticas y de la orientación utilizando indicadores de calidad. Identificar los problemas relativos a la enseñanza y aprendizaje de las materias de la especialización y plantear alternativas y soluciones. Conocer y aplicar metodologías y técnicas básicas de investigación y evaluación educativas y ser capaz de diseñar y desarrollar proyectos de investigación, innovación y evaluación.

Adquirir experiencia en la planificación, la docencia y la evaluación de las materias correspondientes a la especialización. Acreditar un buen dominio de la expresión oral y escrita en la

Practicum práctica docente. Dominar las destrezas y habilidades sociales necesarias para fomentar un clima que facilite el aprendizaje y la

Practicum en la especialización, incluyendo el Trabajo fin de Máster. convivencia. Participar en las propuestas de mejora en los distintos ámbitos de actuación a partir de la reflexión basada en la práctica. Para la formación profesional, conocer la tipología empresarial correspondiente a los sectores productivos y comprender los sistemas organizativos más comunes en las empresas. Respecto a la orientación, ejercitarse en la evaluación (16 créditos europeos) psicopedagógica, el asesoramiento a otros profesionales de la educación, a los estudiantes y a las familias. Estas competencias, junto con las propias del resto de materias, quedarán reflejadas en el Trabajo fin de Máster que compendia la formación adquirida a lo largo de todas las enseñanzas descritas.

Tabla 6. Competencias de cada uno de los módulos que componen el Máster Universitario.

Fuente: Ministerio de Educación, Cultura y Deporte [MECD] (2007). 


\section{Resultados}

En un intento de evaluar el nivel competencial del alumnado que cursa el Máster Universitario que habilita para el ejercicio de la docencia como Profesor/a de Educación Secundaria, la UPO ha recogido una propuesta de valoración de competencias de los futuros docentes durante el curso académico 2014-2015.

En este sentido, se han sintetizado las competencias requeridas en el Máster Universitario en 21 indicadores competenciales clasificados en 5 secciones claves para analizar el grado de adquisición competencial de los estudiantes durante el transcurso del Máster Universitario. En la Tabla 7 se muestra dicha clasificación de indicadores competenciales.

\begin{tabular}{|c|c|c|}
\hline Secciones & Número & $\begin{array}{l}\text { Indicadores competenciales } \\
\end{array}$ \\
\hline \multirow{3}{*}{$\begin{array}{l}\text { Diagnóstico de la } \\
\text { realidad }\end{array}$} & 1 & $\begin{array}{l}\text { Diferencia y recoge datos sobre variables psicológicas, } \\
\text { sociológicas y pedagógicas. }\end{array}$ \\
\hline & 2 & $\begin{array}{l}\text { Comprende la diversidad del aula y los diferentes } \\
\text { ritmos de aprendizaje. }\end{array}$ \\
\hline & 3 & Analiza y define los distintos grupos de destinatarios. \\
\hline \multirow{5}{*}{$\begin{array}{l}\text { Diseño de la } \\
\text { intervención }\end{array}$} & 4 & $\begin{array}{l}\text { Conoce los elementos curriculares a tener en cuenta } \\
\text { para diseñar las clases. }\end{array}$ \\
\hline & 5 & $\begin{array}{l}\text { Conoce la necesidad de contar con la diversidad del } \\
\text { aula para delimitar los objetivos de aprendizaje. }\end{array}$ \\
\hline & 6 & $\begin{array}{l}\text { Plantea una metodología acorde con los objetivos y } \\
\text { los recursos de los que se dispone. }\end{array}$ \\
\hline & 7 & $\begin{array}{l}\text { Secuencia y realiza de modo coherente los contenidos } \\
\text { de una unidad en función de las competencias a } \\
\text { adquirir. }\end{array}$ \\
\hline & 8 & $\begin{array}{l}\text { Plasma todos los elementos curriculares de forma } \\
\text { ordenada y coherente en un diseño de intervención. }\end{array}$ \\
\hline \multirow{8}{*}{ Intervención } & 9 & $\begin{array}{l}\text { Conoce los diferentes modelos de enseñanza y sus } \\
\text { características. }\end{array}$ \\
\hline & 10 & $\begin{array}{l}\text { Analiza el modelo de enseñanza más adecuado para el } \\
\text { correcto desarrollo educativo. }\end{array}$ \\
\hline & 11 & $\begin{array}{l}\text { Utiliza el modelo de enseñanza apropiado para el } \\
\text { correcto desarrollo del aula. }\end{array}$ \\
\hline & 12 & Genera el clima conveniente para el aula. \\
\hline & 13 & Desarrolla una planificación. \\
\hline & 14 & Resuelve una situación imprevista. \\
\hline & 15 & Gestiona el aula frente a situaciones problemáticas. \\
\hline & 16 & $\begin{array}{l}\text { Mantiene la comunicación necesaria con el grupo de } \\
\text { clase y con los compañeros. }\end{array}$ \\
\hline \multirow{4}{*}{ Evaluación } & 17 & Evalúa el contexto educativo. \\
\hline & 18 & $\begin{array}{l}\text { Evalúa los objetivos de aprendizaje a desarrollar en el } \\
\text { aula en base a la secuenciación y selección de } \\
\text { contenidos planificados. }\end{array}$ \\
\hline & 19 & $\begin{array}{l}\text { Evalúa los resultados obtenidos según el grupo de } \\
\text { destinatarios y las capacidades adquiridas. }\end{array}$ \\
\hline & 20 & Evalúa y analizar todos los elementos del currículo. \\
\hline $\begin{array}{l}\text { Propuesta de } \\
\text { innovación }\end{array}$ & 21 & $\begin{array}{l}\text { Expresa coherencia en las propuestas de mejora } \\
\text { presentadas con los datos recogidos en las prácticas. }\end{array}$ \\
\hline
\end{tabular}


A continuación, en la Gráfica 1 se recogen el grado de adquisición competencial de los estudiantes en cada uno de los indicadores anteriores (expresados en \% de estudiantes que lo han adquirido) a lo largo del curso académico 2014-2015.

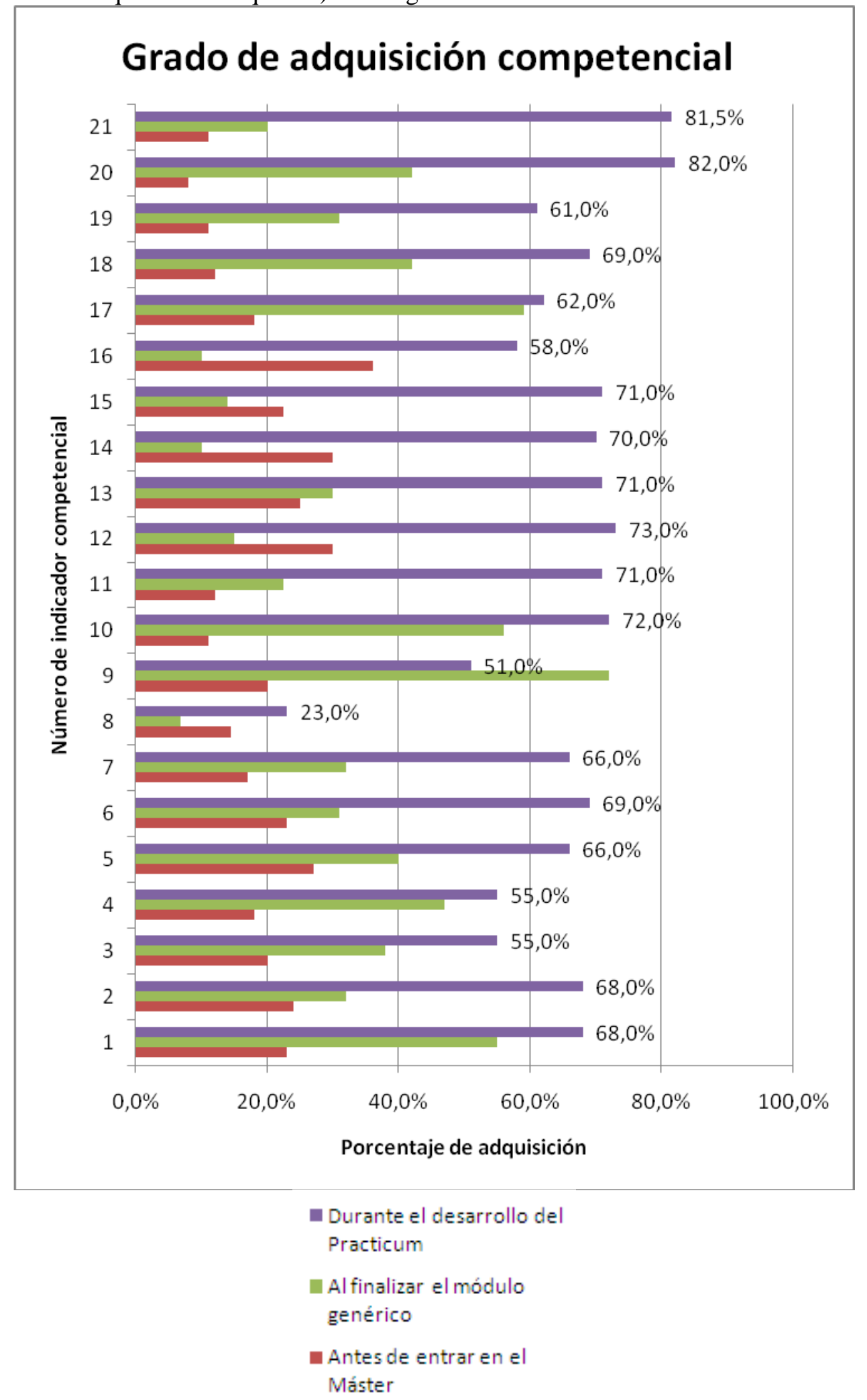

Gráfica 1. Grado de adquisición competencial de los estudiantes en cada uno de los indicadores (expresados en \%) a lo largo del curso académico 2014-15. Fuente. Elaboración propia. 
A la luz de los datos mostrados, se pueden deducir tres conclusiones relevantes:

1. La mayoría de los indicadores competenciales han sido adquiridos progresivamente durante el curso académico 2014-2015 en la UPO. Tan solo los indicadores $8,12,14,15$ y 16 han obtenido un descenso en el grado de adquisición desde el comienzo en el Máster Universitario hasta la finalización del módulo genérico del mismo. Posteriormente, todos estos indicadores señalados aumentaron en su grado de adquisición durante el desarrollo del Practicum.

2. El grado de adquisición de los indicadores competenciales no ha sido uniforme en todos los estudiantes durante el desarrollo del practicum. Así pues, en la Tabla 8 se puede observar que hay 2 indicadores que han superado el $80 \%$ de los alumnos, 14 que se sitúan entre el $60 \%$ y el $80 \%$, y 5 que no llegan al $60 \%$.

\begin{tabular}{cc}
\hline$\%$ alumnos & Número de indicador competencial \\
\hline$\geq 80 \%$ & 20 y 21 \\
\hline$\geq 60 \%$ y $<80 \%$ & $1,2,5,6,7,10,11,12,13,14,15,17,18$ y 19 \\
\hline$<60 \%$ & $3,4,8,9$ y 16 \\
\hline Tabla $8 . \%$ de alumnos que contribuyen a la adquisición de los \\
indicadores competenciales durante el desarrollo del practicum y a lo \\
largo del curso académico 2014-15. \\
Fuente. Elaboración propia.
\end{tabular}

3. El grado de adquisición de los indicadores competenciales antes de entrar en el Máster Universitario era muy bajo en la mayoría de estudiantes, como se muestra en la Tabla 9. Tan solo 1 indicador superaba el $30 \%$ de los estudiantes, 10 se encontraban entre el $20 \%$ y el $30 \%$, y 10 no llegan al $20 \%$.

\begin{tabular}{cc}
\hline \% alumnos & Número de indicador competencial \\
\hline$\geq 30 \%$ & 16 \\
\hline$\geq 20 \% \mathrm{y}<30 \%$ & $1,2,3,5,6,9,12,13,14$ y 15 \\
\hline$<20 \%$ & $4,7,8,10,11,17,18,19,20$ y 21 \\
\hline
\end{tabular}

Tabla 9. \% de alumnos que contribuyen a la adquisición de los indicadores competenciales antes de comenzar el Máster Universitario durante el curso académico 2014-15.

Fuente. Elaboración propia.

\section{Discusión}

En este trabajo se ha realizado una revisión del concepto de competencia a nivel académico y toda la legislación vigente española al respecto en el currículo de la Educación Secundaria. En este sentido, también se ha contemplado los requisitos competenciales de los futuros profesores en su formación inicial y se recoge un diagnóstico del grado de adquisición secuencial de una síntesis de indicadores competenciales basados en los requeridos para este tipo de docencia en España.

Este estudio de caso se ha diseñado en la UPO y durante el periodo académico 2014-2015. Sería necesario realizar un análisis histórico en dicha institución durante los próximos cursos académicos donde se pudiera comparar el grado de adquisición de los niveles competenciales de los futuros profesores en distintos periodos de tiempo y por la misma organización.

Por otro lado, este análisis se ha centrado en una única universidad. Se debería extender la valoración de los indicadores competenciales a todas las universidades españolas que impartan dicho Máster Universitario y a aquellas latino-americanas que posean algún requisito formativo análogo al contemplado en este artículo. 
De esta manera, la escuela y la universidad son los escenarios en los que se mantienen los contextos adecuados para que los futuros profesores de Educación Secundaria adquieran las competencias necesarias para realizarse como grandes profesionales en el mundo educativo. Por tanto, y en base a lo anterior, se hace necesario generar una valoración homologada de indicadores competenciales en todas las instituciones y lo largo del tiempo para que puedan compararse y se produzcan propuestas de mejora continua en la formación inicial de los futuros docentes.

Este recorrido permite acercarse al estudio de las vocaciones de los futuros profesores que van a configurar los centros del mañana, de sus funciones y sus implicaciones en la vida cotidiana de los adolescentes, y por tanto, en la construcción del entramado social necesario en nuestro sistema educativo actual. También permite reconocer a los centros educativos como una construcción histórica que debe responder a las exigencias y movimientos de las realidades sociales mediante profesores competentes.

\section{Conclusiones}

En base a los resultados obtenidos, se deberían tomar medidas preventivas en aquellos indicadores competenciales que no han sido adquiridos progresivamente durante el curso académico 2014-2015. En este sentido, se tendría que proponer para el próximo curso académico algunos ajustes docentes en el Máster Universitario sobre la plasmación de todos los elementos curriculares de forma ordenada y coherente en un diseño de intervención, la generación de un clima conveniente para el aula, la resolución de una situación imprevista, la gestión del aula frente a situaciones problemáticas, y el mantenimiento de la comunicación necesaria con el grupo de clase y con los compañeros.

Por otro lado, se podría analizar las causas de aquellos indicadores competenciales que no han sido superados por el $60 \%$ de los alumnos del Máster Universitario. Es decir, se debería recoger las dificultades de los estudiantes en el análisis y definición de los distintos grupos de destinatarios, el conocimiento de los elementos curriculares a tener en cuenta para diseñar las clases, el establecimiento de todos los elementos curriculares de forma ordenada y coherente en un diseño de intervención, el conocimiento de los diferentes modelos de enseñanza y sus características, y el mantenimiento de la comunicación necesaria con el grupo de clase y con los compañeros.

Y por último, se hace necesario una revisión del perfil de los estudiantes que deciden la elección de la carrera profesional docente en la Educación Secundaria. En los datos analizados anteriormente se concluye que el grado de adquisición de casi todos los indicadores competenciales antes de entrar en el Máster Universitario era muy bajo en la mayoría de usuarios (casi todos se encuentran por debajo del 30\%). No obstante, también hay que reseñar que ese grado de adquisición va progresivamente mejorando y alcanza cotas muy aceptables en la realización del practicum.

\section{Referencias}

Agencia Nacional de Evaluación de la Calidad y Acreditación. (2004). Libro Blanco: Título de Grado en Pedagogía y Educación Social. Volumen 1. Recuperado de http://www.aneca.es/media/150392/libroblanco_pedagogia1_0305.pdf 
Agència per a la Qualitat del Sistema Universitari de Catalunya. (2009). Guía para la evaluación de competencias en el área de Ciencias Sociales. Recuperado de http://www.aneca.es/var/media/150392/libroblanco_pedagogia1 0305.pdf

Domínguez, G. y Hermosilla, J.M. (2010). Propuesta de planificación y reflexión sobre el trabajo docente para el desarrollo de competencias en el EEES. XXI, Revista de Educación, 12, 63-80. Recuperado de http://rabida.uhu.es/dspace/bitstream/handle/10272/5323/b16450097.pdf?sequenc $\underline{\mathrm{e}=2}$

Junta de Andalucía. (2010). Decreto 302/2010, de 1 de julio, por el que se ordena la función pública docente y se regula la selección del profesorado y la provisión de puestos de trabajo docentes. Recuperado de http://www.juntadeandalucia.es/boja/2010/108/2

Junta de Andalucía. (2007). Ley 17/2007, de 10 de diciembre, de Educación de Andalucía. Recuperado de http://www.juntadeandalucia.es/boja/2007/252/1

Junta de Andalucía. (2007). Decreto 93/2013, de 27 de agosto, por el que se regula la formación inicial y permanente del profesorado en la Comunidad Autónoma de Andalucía, así como el Sistema Andaluz de Formación Permanente del Profesorado. Recuperado de http://www.juntadeandalucia.es/boja/2013/170/1

Ministerio de Educación, Cultura y Deporte. (2015). Orden ECD/65/2015, de 21 de enero, por la que se describen las relaciones entre las competencias, los contenidos y los criterios de evaluación de la Educación Primaria, la Educación Secundaria Obligatoria y el Bachillerato. Recuperado de https://www.boe.es/diario_boe/txt.php?id=BOE-A-2015-738

Ministerio de Educación, Cultura y Deporte. (2014). Real Decreto 1105/2014, de 26 de diciembre, por el que se establece el currículo básico de la Educación Secundaria Obligatoria y del Bachillerato. Recuperado de https://www.boe.es/diario boe/txt.php?id=BOE-A-2015-37

Ministerio de Educación, Cultura y Deporte. (2013). Ley Orgánica 8/2013, de 9 de diciembre, para la Mejora de la Calidad Educativa. Recuperado de https://www.boe.es/diario_boe/txt.php?id=BOE-A-2013-12886

Ministerio de Educación, Cultura y Deporte. (2007). Real Decreto 1393/2007, de 29 de octubre, por el que se establece la ordenación de las enseñanzas universitarias oficiales. Recuperado de http://www.boe.es/buscar/act.php?id=BOE-A-200718770

Ministerio de Educación, Cultura y Deporte. (2007). Real Decreto 276/2007, de 23 de febrero, por el que se aprueba el Reglamento de ingreso, accesos y adquisición de nuevas especialidades en los cuerpos docentes a que se refiere la Ley Orgánica 2/2006, de 3 de mayo, de Educación, y se regula el régimen transitorio de ingreso a que se refiere la disposición transitoria decimoséptima de la citada ley. Recuperado de http://www.mecd.gob.es/servicios-al-ciudadano-mecd/catalogoservicios/empleo-publico/funcionario-docente/oposiciones/normativa.html 
Ministerio de Educación, Cultura y Deporte. (2007). Orden ECI/3858/2007, de 27 de diciembre, por la que se establecen los requisitos para la verificación de los títulos universitarios oficiales que habiliten para el ejercicio de las profesiones de Profesor de Educación Secundaria Obligatoria y Bachillerato, Formación Profesional y Enseñanzas de Idiomas. Recuperado de https://www.boe.es/diario boe/txt.php?id=BOE-A-2007-22450

Ministerio de Educación, Cultura y Deporte. (2006). Ley Orgánica 2/2006, de 3 de mayo, de Educación. Recuperado de http://www.boe.es/buscar/act.php?id=BOE$\underline{\text { A-2006-7899 }}$

Unión Europea. (2006). Recomendación 2006/962/CE del Parlamento Europeo y del Consejo, de 18 de diciembre de 2006, sobre las competencias clave para el aprendizaje permanente. Recuperado de http://www.mcu.es/cine/docs/Novedades/Recomendacion_Parlamento_Europeo_ Consejo_Aprendizaje_permanente.pdf 